\title{
Abbreviated protocol combining quantitative diffusion-weighted imaging: a new strategy increasing diagnostic accuracy for breast magnetic resonance imaging?
}

\author{
Zhenzhen Shao, Peifang Liu, Shuping Zhang, Hong Lu \\ Department of Breast Imaging, Tianjin Medical University Cancer Institute, and Hospital, National Clinical Research Center for Cancer, Key \\ Laboratory of Breast Cancer Prevention and Therapy, Tianjin Medical University, Ministry of Education, Key Laboratory of Cancer Prevention and \\ Therapy, Tianjin, China \\ Contributions: (I) Conception and design: H Lu, Z Shao; (II) Administrative support: P Liu; (III) Provision of study materials or patients: Z Shao; (IV) \\ Collection and assembly of data: Z Shao, S Zhang; (V) Data analysis and interpretation: Z Shao; (VI) Manuscript writing: All authors; (VII) Final \\ approval of manuscript: All authors. \\ Correspondence to: Hong Lu, MD. Huan Hu Xi Road, Ti Yuan Bei, He Xi District, Tianjin 300060, China. Email: honglu@tmu.edu.cn.
}

Background: To compare the diagnostic accuracy of an abbreviated protocol (AP) with or without quantitative apparent diffusion coefficient (ADC) values on diffusion-weighted imaging (DWI) and a full diagnostic protocol (FDP) in terms of the Breast Imaging Reporting and Data System (BI-RADS) classification of breast magnetic resonance imaging (MRI).

Methods: Our study sample consisted of 436 patients undergoing breast MRI from January to October 2015 in a clinical setting. The three reviews included a pre-contrast and the first single post-contrast T1weighted (T1W) sequences (AP1), AP1 combined with quantitative DWI (AP2), and the FDP, the AP1 of which were assessed independently by a junior and senior radiologist. Agreement on the evaluation of the BIRADS classifications (between the junior and senior radiologists, between AP1 and FDP, and between AP2 and FDP) was assessed using the kappa test statistic. Sensitivity, specificity, positive predictive value (PPV), and negative predictive value (NPV) were compared between AP1 and FDP plus between AP2 and FDP. Diagnostic parameters of these reviews were examined using the McNemar test.

Results: The study included 436 patients, with 251 breast cancers, 99 benign lesions, and 86 patients with benign or no lesions and followed up for at least 24 months. The agreement of the BI-RADS classifications between the junior and senior radiologists was very good (kappa =0.847). The agreement between AP2 and FDP (kappa =0.931) was higher than the agreement between AP1 and FDP (kappa $=0.872)$ on evaluating the BI-RADS benign and malignant classifications. The sensitivity/specificity/PPV/NPV was 95.6\%/83.8\%/88.9\%/93.4\% for AP1, 98.0\%/83.8\%/89.1\%/96.9\% for AP2, $98.8 \% / 83.8 \% / 89.2 \% / 98.1 \%$ for FDP, respectively.

Conclusions: The addition of quantitative DWI to the abbreviated MRI protocol based on the pre-and first post-contrast sequence improved diagnostic performance for characterizing breast lesions. Quantitative DWI may be a useful adjunct to dynamic contrast enhancement (DCE) of breast MRI.

Keywords: Breast cancers; magnetic resonance imaging (MRI); diffusion-weighted images; abbreviated protocol (AP)

Submitted Jun 09, 2021. Accepted for publication Aug 30, 2021.

doi: $10.21037 / g s-21-463$

View this article at: https://dx.doi.org/10.21037/gs-21-463

(c) Gland Surgery. All rights reserved. 


\section{Introduction}

Breast magnetic resonance imaging (MRI) is the most sensitive imaging technique for detecting breast cancer because it is not affected by breast density. MRI plays an important role in screening women at high risk of developing breast cancer, but it takes about 30 to 40 minutes to scan a breast MRI, limiting its wider use. The long acquisition times not only increase cost but also cause patient discomfort.

Many recent studies have used an abbreviated protocol (AP) to make MRI more accessible while maintaining diagnostic accuracy. Kuhl et al. (1) were the first to have addressed the concept of an AP of breast MRI in Germany in 2014. These authors found that an AP could achieve an equivalent diagnostic accuracy and cancer yield compared to a full diagnostic protocol (FDP) in a sample of 443 highrisk women. Mango et al. (2) found high sensitivity of an AP to detect cancers, but the MIP (Max -imum Intensity Projection) alone may be fewer than the FDP in the detection of breast cancer. Heacock et al. (3) found that there was no effect on the cancer detection rate with the addition of the T2-weighted (T2W) sequence, but lesion conspicuity was significantly increased. These studies have performed various MRI APs, including fat-suppressed first T1-weighted (T1W) post-contrast, subtraction MIP, and fat-saturated pre-contrast $\mathrm{T} 2 \mathrm{~W}$, but few studies have evaluated the accuracy of the diffusion-weighted imaging (DWI) sequence. DWI is relatively quick and easy to implement. It is based on the thermal motion of water molecules in the breast tumor tissue and provides information related to cancer cells and the integrity of cell membranes. A quantitative value, the apparent diffusion coefficient (ADC) measured for DWI, represents the complex diffusion of water in breast tissues (4). Two meta-analyses evaluating the diagnostic performance of quantitative breast DWI demonstrated overall better specificity than dynamic contrast enhancement (DCE) MRI $(5,6)$. Horvat et al. $(7)$ evaluate that different ADC metrics by drawing a region of interest on ADC maps on the whole tumor (WTu) and on its darkest part (DpTu) for prediction of receptor status, proliferation rate, molecular subtype in breast cancer, which found that maximum WTu ADC values may be used to differentiate luminal from other molecular subtypes. Recent studies indicate that the ability to detect breast cancers of an AP based on DWI could be compared to an AP based on post-contrast MRI (8).

Thus, this study aimed to retrospectively compare the diagnostic accuracy of an AP with and without quantitative DWI to that of an FDP in terms of the Breast Imaging Reporting and Data System (BI-RADS) classifications of breast MRIs. Moreover, we evaluated the variability of different breast cancer characteristics. We present the following article in accordance with the STARD reporting checklist (available at https://dx.doi.org/10.21037/gs-21-463).

\section{Methods}

\section{Patients}

From January to October 2015, we retrospectively enrolled 436 consecutive patients who underwent breast MRI at our hospital. All procedures performed in this study involving human participants were in accordance with the Declaration of Helsinki (as revised in 2013). The study was approved by institutional ethics board of Tianjin Medical University Cancer Institute and Hospital (No. bc2021112). Individual consent for this retrospective analysis was waived. Inclusion criteria were pathologic features or more than 24 months of follow-up. Patients undergoing neoadjuvant chemotherapy were excluded. The mean age of the 436 patients was 42.5 years old (range, 17-74 years). Of the 436 patients, 251 cases had malignant lesions and 99 cases had benign lesions by confirmed pathologically, and the remaining 86 cases were benign with at least 24 months of followup. Interpretations were performed in three review stages as follows: (I) AP1 consisted of a pre-contrast and the first single post-contrast T1W sequence; (II) AP2 was the AP1 combined with the ADC value measured on axial DWI; and (III) the FDP included all of the sequences. Table 1 lists the sequences for the three study protocols.

Two readers (a junior and a senior radiologist with 5 and

Table 1 Comparisons of the two abbreviated protocols on breast magnetic resonance imaging

\begin{tabular}{lcccc}
\hline Protocol & $\begin{array}{c}\text { Dynamic } \\
\text { pre-contrast }\end{array}$ & $\begin{array}{c}\text { Dynamic first } \\
\text { post-contrast }\end{array}$ & $\begin{array}{c}\text { Axial diffusion-weighted } \\
\text { imaging (DWI) }\end{array}$ & $\begin{array}{c}\text { Apparent diffusion } \\
\text { coefficient (ADC) value }\end{array}$ \\
\hline AP1 & $\sqrt{ }$ & $\sqrt{ }$ & \\
AP2 & $\sqrt{ }$ & $\sqrt{ }$ & $\sqrt{ }$ \\
\hline
\end{tabular}


10 years of breast MRI experience, respectively) individually read the images in the AP with randomization to limit all bias. If the BI-RADS classifications and the two readers' lesion location were found to be discordant, the consensus was sought through discussion. For each breast, the readers indicated the quadrant, lesion size, and the American College of Radiology (ACR) BI-RADS classification. For the review stages of the AP2 and FDP, the senior physician evaluated the ACR BI-RADS classification. The BI-RADS classifications were then dichotomized to evaluate diagnostic accuracy: BI-RADS 1, 2, 3, and 4A were considered negative, and BI-RADS 4B, 4C, and 5 were considered negative positive.

\section{MRI technique}

The full diagnostic MRI examinations were performed using a 3.0 T magnet (GE Discovery 750, GE Healthcare, Chicago, IL, USA ) with patients in the prone position using a dedicated breast coil. The breasts were imaged with an axial T1W sequence (repetition time, TR $=622 \mathrm{~ms}$, echo time, $\mathrm{TE}=10 \mathrm{~ms}$ ), a fat-suppressed axial $\mathrm{T} 2 \mathrm{~W}$ sequence ( $\mathrm{TR}=6,330 \mathrm{~ms}$, $\mathrm{TE}=68 \mathrm{~ms}$ ), and an axial $\mathrm{DWI}$ sequence $(\mathrm{TR}=3,235 \mathrm{~ms}$, TE $=64 \mathrm{~ms}$ ) with $\mathrm{b}$ values of 0 and $1,000 \mathrm{~s} / \mathrm{mm}^{2}$. The contrast enhancement scan used the sagittal T1W fat-suppressed VIBRANT (volume imaging for breast assessment $)$ sequence $(\mathrm{TR}=6.1 \mathrm{~ms} ; \mathrm{TE}=2.9 \mathrm{~ms}$ : flip angle $=15^{\circ}$; matrix $=256 \times 128$ : slice thickness $=1.8 \mathrm{~mm}$; phase acquisition time $=90-100 \mathrm{~s}$ ), obtained before and continuous 5 times after intravenous injection of $0.1 \mathrm{mmol} / \mathrm{L}$ gadolinium chelate per kilogram of body weight (Gadovist, Bayer Schering Pharma, Berlin, Germany). The AP included the sagittal VIBRANT sequence before injection and the first sagittal VIBRANT sequence after injection.

\section{Statistical analysis}

Agreement on the evaluation of the BI-RADS classifications (between the junior and senior radiologist, between AP1 and FDP, and between AP2 and FDP) was assessed using the kappa statistic. Kappa values were interpreted as follows: less than 0.20 indicates poor agreement; $0.21-0.40$ indicates fair agreement; $0.41-0.60$ indicates moderate agreement; $0.61-0.80$ indicates good agreement; and $0.81-1.00$ indicates very good agreement. The sensitivity, specificity, positive predictive value (PPV), and negative predictive value (NPV) for each protocol were computed, comparing AP1 with FDP and AP2 with FDP using McNemar's test. The
Statistical Program for the Social Sciences (SPSS) software (version 17.0) was used to perform the statistical analyses, and $\mathrm{P}<0.05$ indicated statistical significance.

\section{Results}

\section{Distribution of BI-RADS classifications in the three different protocols}

We examined the distribution of BI-RADS classifications AP1, AP2, and FDP. The number of category BI-RADS $1-4 \mathrm{~A}$ was 166 of 436 (38.1\%) by using AP1, 160 of 436 (36.7\%) by using AP2, and 158 of 436 (36.2\%) by using FDP. For the category BI-RADS 4B-5, AP1 for breast MRI classified 61.9\% (270/436), AP2 classified 63.3\% (276/436), and FDP classified 63.8\% (278/436) (Figure 1).

\section{Inter-reader and inter-protocol concordance analyses}

Figure 2 shows a comparison of the evaluations of the BIRADS classifications for AP1 between the junior and senior radiologists. The agreement of BI-RADS classifications between the junior and senior radiologists was very good (kappa $=0.847)$. Although there was very good inter-reader concordance, we pooled the BI-RADS classifications for AP 1 of the two readers. Then we evaluated the classifications for AP2. The agreement between AP2 and FDP (kappa $=0.931$ ) was higher than the agreement between AP1 and FDP (kappa $=0.872$ ) on evaluating the BIRADS benign and malignant classifications.

\section{Diagnostic efficiency for the three different protocols}

Of the 436 women in our study, 251 breast cancers and 99 benign lesions were confirmed based on pathological characteristics. The reference standard was assessed by a follow-up of more than 24 months for 86 women with benign or no breast lesions. Of the 251 cancers, 221 lesions were invasive carcinomas, 22 were ductal carcinomas in situ (DCIS), 6 were invasive lobular carcinomas, and 1 case each was malignant phyllodes and an angiosarcoma. The 159/221 $(71.9 \%)$ invasive cancers were intermediate nuclear grade, and the majority of DCIS (13/22, 59.1\%) were intermediate and high nuclear grade. Invasive cancers were 32/221 (14.6\%) luminal-A subtype, 141/221 (63.8\%) luminal-B subtype, 26/221 (11.8\%) HER2-enriched subtype, and 22/221 (10.0\%) triple-negative breast cancers.

The accuracy to detect a cancer was not significantly 


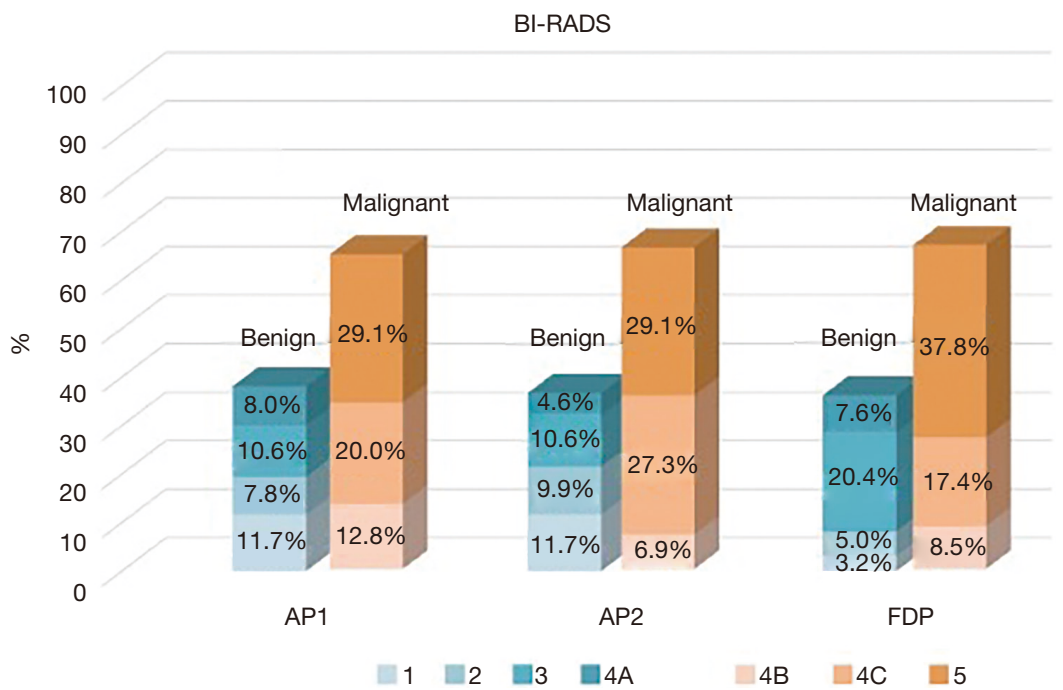

Figure 1 Distribution of BI-RADS classifications in three different protocols. BI-RADS, Breast Imaging Reporting and Data System.

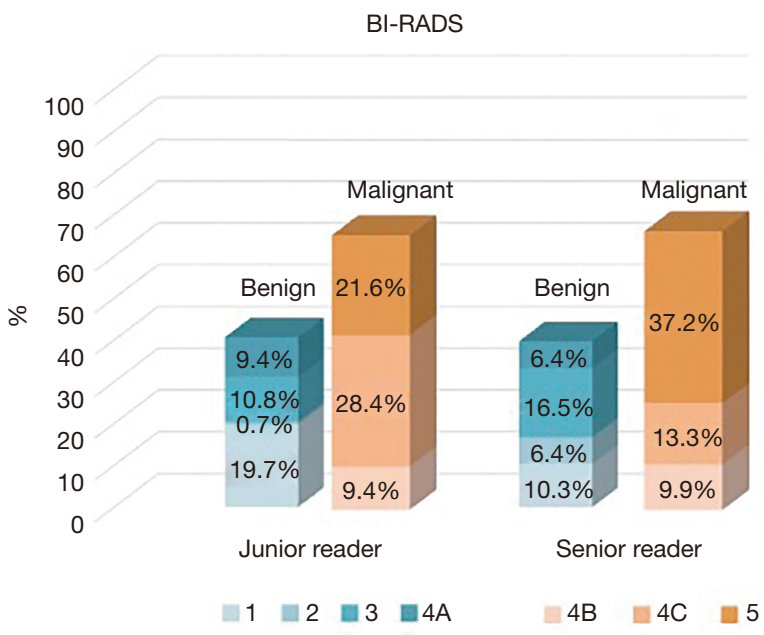

Figure 2 Distribution of BI-RADS classifications in junior and senior reader. BI-RADS, Breast Imaging Reporting and Data System.

different between the two protocols: $90.6 \%$ (395/436) of AP1 vs. $92.4 \%(403 / 436)$ of FDP, $\mathrm{P}=0.331$; and $92.0 \%$ (401/436) of AP2 vs. 92.4\% (403/436) of FDP, $\mathrm{P}=0.801$. Of the 251 cancers, 240 were classified as "BI-RADS 4B-5" for AP1 versus 248 for FDP, and the sensitivity was $95.6 \%$ and $98.8 \%$, respectively. For AP2, the sensitivity was $98.0 \%$ (246/251), which was not significantly different between the AP2 and FDP $(\mathrm{P}=0.476)$, but there was a trend towards statistical significance in sensitivity between AP1 and FDP $(\mathrm{P}=0.054)$. The sensitivity of AP2 was higher than that of
$\mathrm{AP} 1$, indicating that more positive findings were detected using the AP with combined quantitative DWI. Out of the 185 benign breast lesions, 155 were classified as "BI-RADS $1-4 \mathrm{~A}$ " using the three different protocols (specificity, $83.8 \%$ ) (Table 2). The PPV/NPV were $88.9 \%(240 / 270) / 93.4 \%$ (155/166) for AP1, 89.1\% (246/276)/96.9\% (155/160) for AP2, and $89.2 \%(248 / 278) / 98.1 \%(155 / 158)$ for FDP respectively. There were no significant differences in PPV and NPV between protocols (Table 2).

\section{Pathologic characteristics and breast MRI features of missed cancers}

For AP1, 11 breast cancers were missed. Five and 3 carcinomas were missed for AP2 and FDP, respectively. About half $(45.5 \%, 5 / 11)$ of missed cancers were foci enhancement, the 3 missed mass-enhancement cancers had a tumor size of less than $2 \mathrm{~cm}$, and 2 of the other 3 nonmass-enhancement cancers were DCIS. Five out of 11 (45.5\%) of missed cancers were DCIS, and 6/11 (54.5\%) were intermediate-grade invasive ductal cancers. Of the 5 DCIS, 4 cases were low grade and one intermediate grade. Of the 6 invasive ductal cancers, the largest missed cancer (size $6 \mathrm{~cm}$, pathologic stage T3) was a nonmass enhancement cancer on MRI with marked BPE (Background Parenchymal Enhancement), and other missed cancers were pathologic stage T1. The majority (10/11, $90.9 \%$ ) of missed cancers were of a luminal subtype (Table 3). Magnetic resonance images of the two missed cancers are 
Table 2 Diagnostic efficiency for the three different protocols

\begin{tabular}{lccccc}
\hline & Accuracy & Sensitivity & Specificity & PPV & NPV \\
\hline AP1 vs. FDP & $90.6 \%$ vs. $92.4 \%$ & $95.6 \%$ vs. $98.8 \%$ & $83.8 \%$ vs. $83.8 \%$ & $88.9 \%$ vs. $89.2 \%$ & $93.4 \%$ vs. $98.1 \%$ \\
& $(\mathrm{P}=0.331)$ & $(\mathrm{P}=0.054)$ & $(\mathrm{P}=1.000)$ & $(\mathrm{P}=0.905)$ & $(\mathrm{P}=0.053)$ \\
AP2 vs. FDP & $92.0 \%$ vs. $92.4 \%$ & $98.0 \%$ vs. $98.8 \%$ & $83.8 \%$ vs. $83.8 \%$ & $89.1 \%$ vs. $89.2 \%$ & $96.9 \%$ vs. $98.1 \%$ \\
& $(\mathrm{P}=0.801)$ & $(\mathrm{P}=0.476)$ & $(\mathrm{P}=1.000)$ & $(\mathrm{P}=0.976)$ & $(\mathrm{P}=0.485)$ \\
\hline
\end{tabular}

PPV, positive predictive value; NPV, negative predictive value.

Table 3 Pathologic characteristics and breast MRI features of missed cancers

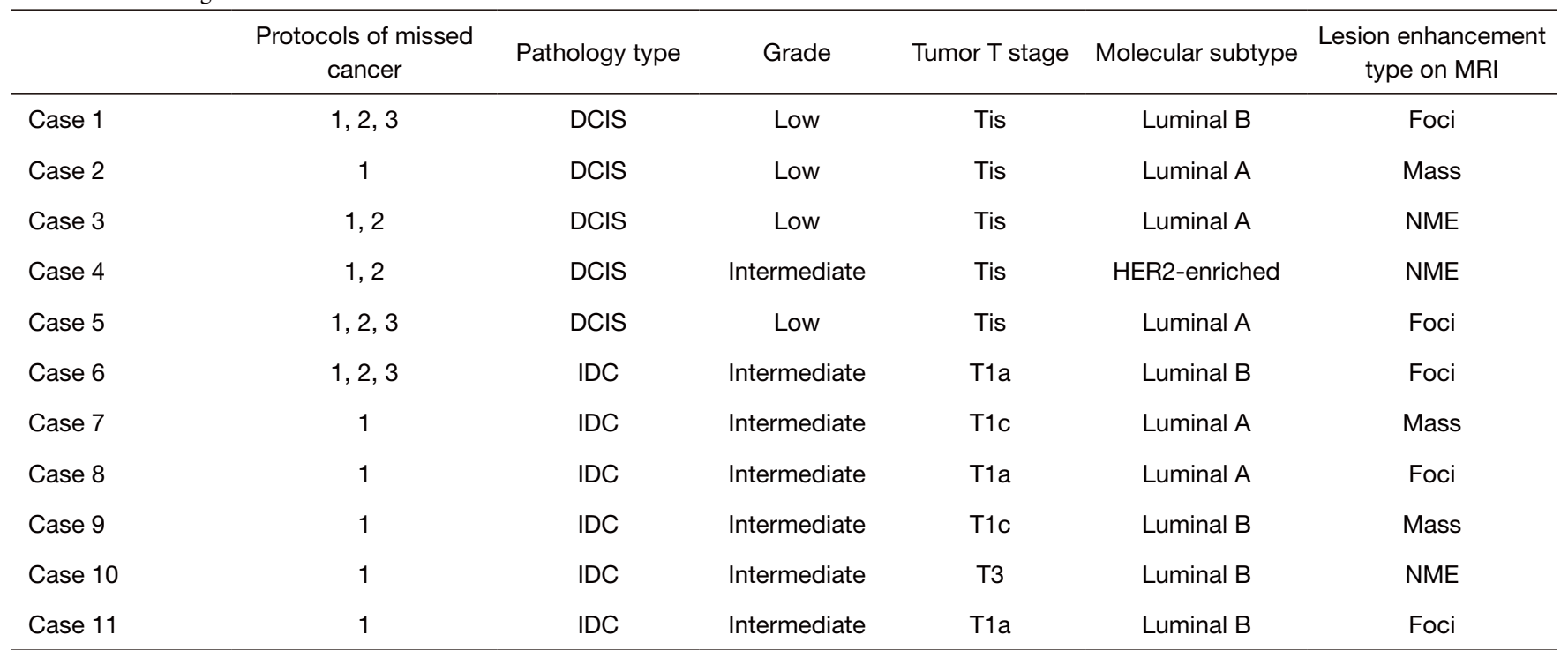

1, AP1 missed; 2, AP2 missed; 3, FDP missed. IDC, invasive ductal carcinoma; DCIS, ductal carcinoma in situ; MRI, magnetic resonance imaging; NME, non-mass enhancement; Tis, ductal carcinoma in situ is Tis stage in pathology.

shown in Figures 3,4.

\section{Detection of breast cancers with different MRI enhancement features for the three different protocols}

Of the 251 breast cancers, $5(2.0 \%)$ were focus enhancements, $165(65.7 \%)$ were mass enhancements, and 81 (32.3\%) were non-mass enhancements. For AP1, there was no detection of breast cancers presenting as focus enhancements, and there was a detection of $98.2 \%$ of breast cancers presenting as mass enhancements, and $96.3 \%$ of breast cancers presenting as non-mass enhancements. For AP2, after the addition of DWI and ADC values, 60\% (3/5) of cancers showing focus enhancement were detected, all cancers of mass enhancement were detected, and $97.5 \%$ (79/81) of cancers showing nonmass enhancement were detected. For the FDP, all cancers of mass- and non-mass enhancements were detected, and $60 \%$ $(3 / 5)$ of cancers representing as focus enhancements were detected (Figure 5).

\section{Discussion}

Our study showed that the AP's accuracy was high and not significantly different from the FDP. The accuracy of the $\mathrm{AP}$ with an $\mathrm{ADC}$ value was higher than the $\mathrm{AP}$ without an $\mathrm{ADC}$ value and similar to that of the FDP.

For the AP1, there was a very good inter-reader agreement between the junior and senior radiologists. The use of the AP was hence reproducible in the interpretation, and the ensuing follow-up. A pooled BI-RADS classification of the AP1 was obtained from two readers. There was also nearly complete concordance (above 0.80 ) between the AP1 and FDP and between the AP2 and FDP. The BIRADS classification of AP2 was closer to the FDP than AP1. Moreover, the three protocols had high sensitivity and specificity, and AP1 and AP2 did not influence the 

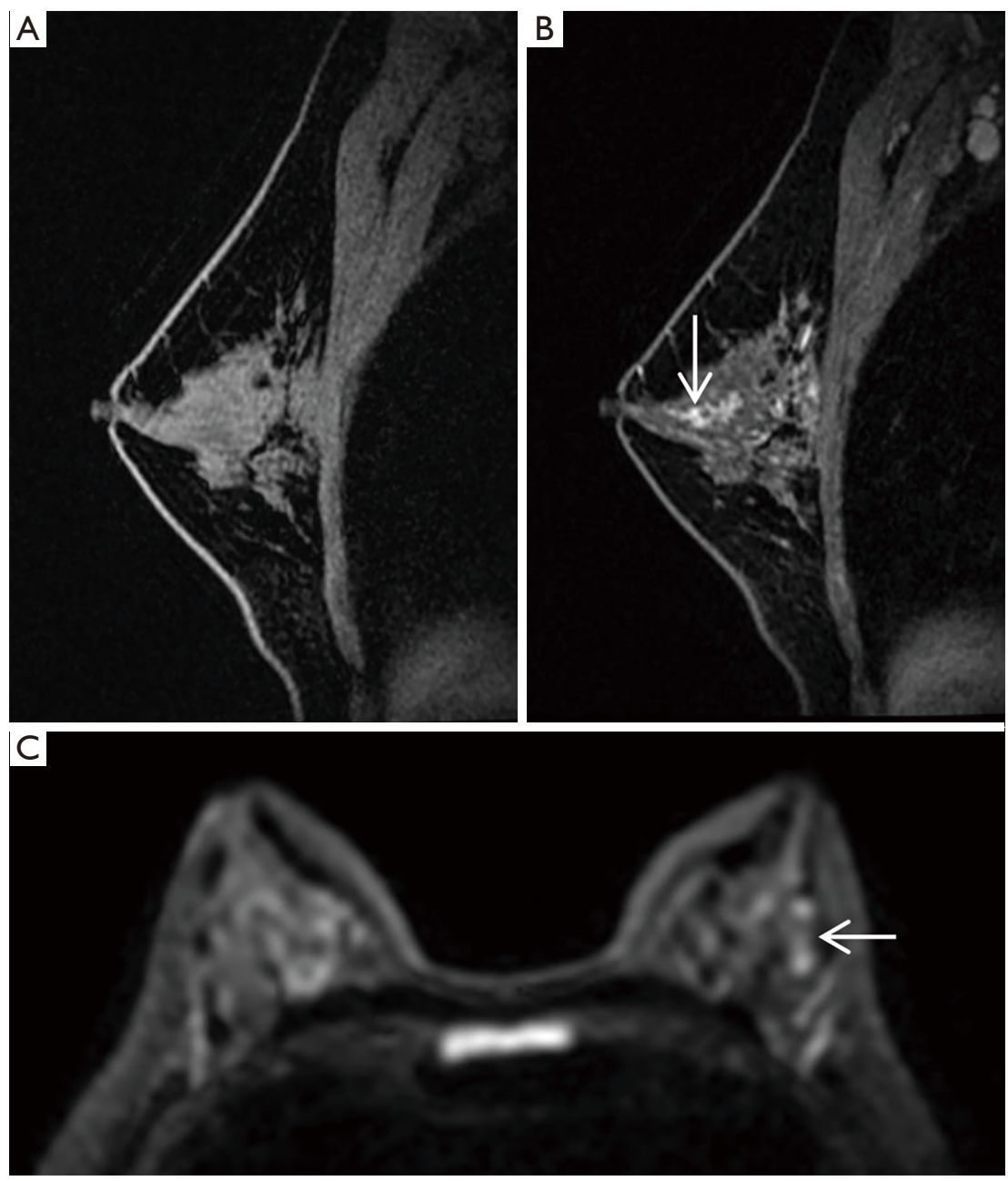

Figure 3 A 31-year-old woman with left nipple discharge. Cancer appearing as non-mass enhancement was missed on AP1 and AP2. Sagittal T1-weighted fat-suppressed pre-contrast VIBRANT sequence (A), sagittal T1-weighted fat-suppressed first post-contrast VIBRANT sequence (B), and diffusion-weighted sequence $\left(b=1,000 \mathrm{~s} / \mathrm{mm}^{2}\right)(\mathrm{C})$. Magnetic resonance images of the left breast show a ductal enhancing non-mass (arrows) at image (B). There was no corresponding increased signal on diffusion-weighted imaging, and the apparent diffusion coefficient value was $1.53 \times 10^{-3} \mathrm{~mm}^{2} / \mathrm{s}$. Biopsy yielded low-grade ductal carcinoma in situ.

diagnostic accuracy of the examination. The addition of DWI and ADC values led to higher sensitivity than the sequence that only included pre-contrast and first postcontrast T1WI, and the addition of DWI and ADC values did not compromise specificity. Kuhl et al. (1) first demonstrated that an AP breast MRI is feasible for breast screening by MRI. Mango et al. (2) demonstrated that an AP had high sensitivity for detection of cancers and that the mean sensitivity was $96 \%$ for the first post-contrast, $96 \%$ for the first post-contrast subtraction, and $93 \%$ for the subtraction MIP. These reported studies mainly focused on breast cancer screening using an abbreviated magnetic resonance $(\mathrm{MR})$ protocol $(1,2,9)$. In contrast to these reported series, Moschetta et al. (10) analyzed the diagnostic accuracy of an AP combining the morphological magnetic resonance sequences (short time inversion recovery, T2weighted turbo spin echo ) for characterizing breast lesions and found the same diagnostic capacity as for the FDP. It could be used not only for cancer screening but also for MR breast lesion characterization. Similarly, Heacock et al. (3) showed that the addition of a T2-weighted sequence did not affect cancer detection, but T2-imaging significantly improved lesion conspicuity.

In contrast to the reported series, the AP in this study 

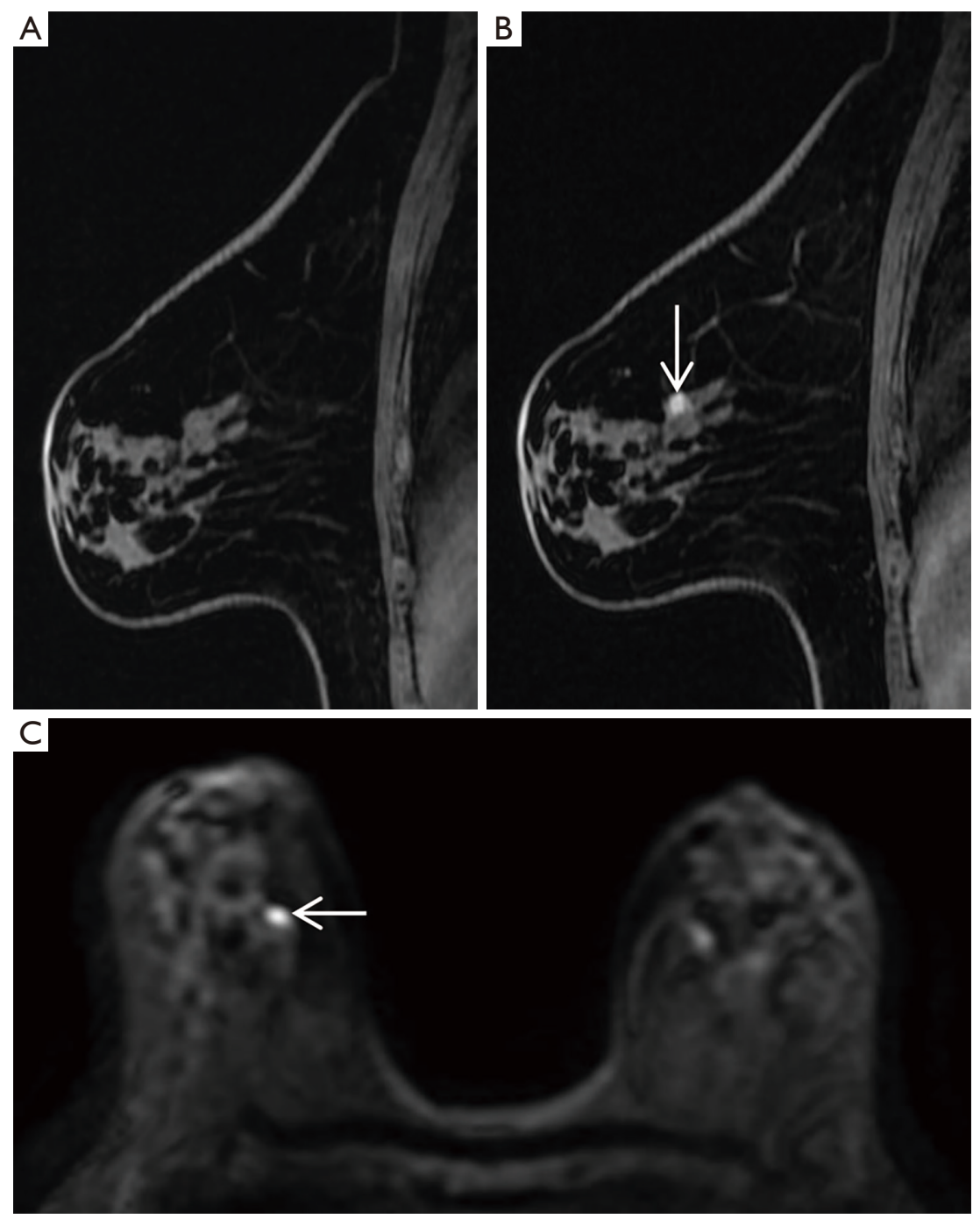

Figure 4 A 55-year-old woman with a mass in the upper inner quadrant of the right breast. Cancer appearing as focus enhancement was missed on AP1. Sagittal T1-weighted fat-suppressed pre-contrast VIBRANT sequence (A), sagittal T1-weighted fat-suppressed first postcontrast VIBRANT sequence (B), and diffusion-weighted sequence (b=1,000 s/mm $\mathrm{m}^{2}$ (C). Magnetic resonance images of the left breast show a ductal enhancing non-mass (arrows) at image (B). There was a corresponding increased focus signal on diffusion-weighted imaging, and the apparent diffusion coefficient value was $0.96 \times 10^{-3} \mathrm{~mm}^{2} / \mathrm{s}$. Biopsy yielded intermediate-grade invasive ductal carcinoma.

included the pre-contrast and the first post-contrast T1WI sequence, in addition to the quantitative DWI in order to characterize breast benign or malignant lesions. To our knowledge, Yamada et al. (8) compared the performance of two APs, including an AP1 based on DWI and an AP2 based on DCE-MRI. This study was focused on breast cancers $\leq 2 \mathrm{~cm}$ in diameter and found that the detectability of an unenhanced AP based on DWI was comparable to that of an AP based on post-contrast MRI and that the accuracy was not significantly different between protocols.
In addition to a DWI protocol, we measured an ADC value for characterizing breast lesions in our series. We found that obtaining an ADC value was frequently a useful adjunct to DCE. DWI effectively distinguishes malignant from benign lesions in patients with clinically suspicious and known findings (10). In our study, the sensitivity was improved from 95.6\% without an ADC to $98.0 \%$ with an ADC, the PPV was improved from $88.9 \%$ without an ADC to $89.1 \%$ with an ADC, and the NPV was improved from $93.4 \%$ without an ADC to $96.9 \%$ with an ADC. Bickelhaupt et al. (11) 

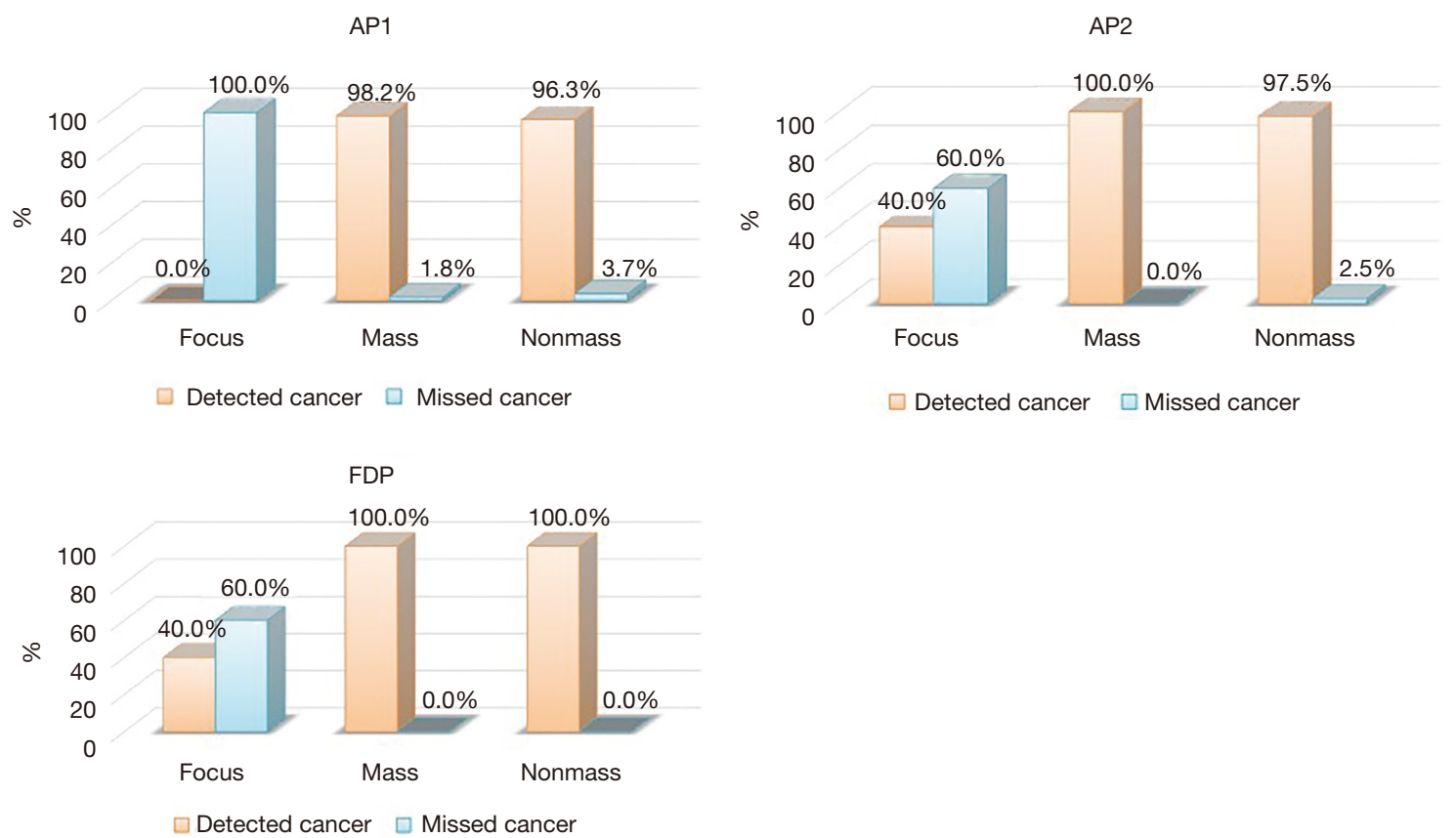

Figure 5 Detection of breast cancers with different magnetic resonance imaging enhancement features for the three different protocols.

compared the diagnostic efficiency of two APs using patients with suspicious findings on a mammogram. The sensitivity and specificity were $92 \%$ and $94 \%$ for the AP based on DWI and $85 \%$ and $90 \%$ for the AP based on post-contrast MRI. The sensitivity of the AP2 (with an ADC value) in our study was similar to that reported by Bickelhaupt et al., but the specificity was low. We compared the diagnostic abilities of the first post-contrast T1WI with an ADC value and the first post-contrast T1WI without an ADC value, but we did not evaluate the diagnostic abilities if imaging was only based on DWI.

In our study, the radiologists missed more lesions with $\mathrm{AP} 1$ (without $\mathrm{ADC}$ value) than with AP2 (with ADC value), but the lesion detectability did not differ significantly. For AP1, almost half of the missed tumors were DCIS, of which $80 \%$ were low grade. For AP1, the missed 6 invasive ductal carcinomas (IDCs) ranged in size from 0.5 to $1.4 \mathrm{~cm}$, in addition to 1 missed IDC, which measured $6 \mathrm{~cm}$ and was a non-mass enhancement on MRI with marked BPE. For AP2, most of the missed tumors were DCIS, and only 1 missed one was an IDC measuring $0.5 \mathrm{~cm}$. This indicates that IDC lesions missed for the AP2 (with an ADC value) were fewer than for the AP1 (without an ADC value), and the missed DCIS lesions were comparable regardless of the presence of an ADC value. This was probably because DWI has a low resolution for foci lesion and low conspicuity for DCIS. Yamada et al. (8) showed that DWI has a lower resolution than post-contrast MRI. In our study, the missed DCIS lesions were almost all low grade. Missing DCIS lesions are inevitable. A recent study indicates that surgical treatment alone does not improve the prognosis of low-grade DCIS $(12,13)$, that women who carry a BRCA1 mutation do not develop DCIS $(14,15)$, and that MRI cannot definitively differentiate low-grade DCIS from intermediate- or highgrade DCIS on MRI (16) However, Lehotska et al. (17) found that DWI and multi-parametric analyses provide important information about the degree of nuclear grade. Furthermore, our readers missed an intermediate-grade DCIS with HER2-enriched subtype, demonstrating scope for further improvement in DCIS detection on MRI. In our study, almost all cancers presenting as mass- and non-mass enhancements were discovered. However, it is important to note that missed cancers mainly appeared as focus enhancements on breast MRI; in particular, all cancers appearing as focus enhancements were missed on the AP1, however, two T1a breast cancers appearing as focus enhancements were detected for AP2 by increasing $\mathrm{ADC}$ value. This was probably because the morphological characteristics have low conspicuity for foci lesions on first post-contrast enhancement imaging. Hence, it could be that 
detection of cancers appearing as focus enhancements are more challenging with an AP. Heacock et al. (3) found that $60 \%$ of missed cancers were non-mass, $60 \%$ were invasive ductal carcinoma, and $60 \%$ were low-grade. Hence, it is able to improve the detection rate for breast cancers presenting as focus enhancement by increasing $\mathrm{ADC}$ value.

Our study has several limitations. First, all patients were enrolled through a hospital clinic. This sample composition would have influenced the detection of positive findings, because of the distributions of age, disease characteristics, and the intensity of the background parenchyma were quite different from the screening setting. Second, we did not compare the reading time among AP1, AP2, and FDP. ADC value measurement is time-consuming, and hence the radiologists took more time to read the images of AP2. Third, our study did not separately analyze the influence of the factors of body mass index, family history, and background enhancement, which might be of interest for future studies.

\section{Conclusions}

In conclusion, an AP combining per- and first postcontrast sequence and quantitative DWI have the same diagnostic efficiency as the full protocol for characterizing breast lesions. Quantitative DWI was a relatively quick, useful adjunct to DCE of breast MRI. The abbreviated MR protocol based on the first post-contrast sequence and quantitative DWI could reduce patient discomfort and increase the number of examinations, not only for breast cancer screening and MR breast lesion characterization.

\section{Acknowledgments}

Funding: Chinese National Key Research and Development Project (Grant number 2018YFC1315601).

\section{Footnote}

Reporting Checklist: The authors have completed the STARD reporting checklist. Available at https://dx.doi. org/10.21037/gs-21-463

Data Sharing Statement: Available at https://dx.doi. org/10.21037/gs-21-463

Conflicts of Interest: All authors have completed the ICMJE uniform disclosure form (available at https://dx.doi. org/10.21037/gs-21-463). The authors have no conflicts of interest to declare.

Ethical Statement: The authors are accountable for all aspects of the work in ensuring that questions related to the accuracy or integrity of any part of the work are appropriately investigated and resolved. All procedures performed in this study involving human participants were in accordance with the Declaration of Helsinki (as revised in 2013). The study was approved by institutional ethics board of Tianjin Medical University Cancer Institute and Hospital (No.: bc2021112). Individual consent for this retrospective analysis was waived.

Open Access Statement: This is an Open Access article distributed in accordance with the Creative Commons Attribution-NonCommercial-NoDerivs 4.0 International License (CC BY-NC-ND 4.0), which permits the noncommercial replication and distribution of the article with the strict proviso that no changes or edits are made and the original work is properly cited (including links to both the formal publication through the relevant DOI and the license). See: https://creativecommons.org/licenses/by-nc-nd/4.0/.

\section{References}

1. Kuhl CK, Schrading S, Strobel K, et al. Abbreviated breast magnetic resonance imaging (MRI): first postcontrast subtracted images and maximum-intensity projection-a novel approach to breast cancer screening with MRI. J Clin Oncol 2014;32:2304-10.

2. Mango VL, Morris EA, David Dershaw D, et al. Abbreviated protocol for breast MRI: are multiple sequences needed for cancer detection? Eur J Radiol 2015;84:65-70.

3. Heacock L, Melsaether AN, Heller SL, et al. Evaluation of a known breast cancer using an abbreviated breast MRI protocol: Correlation of imaging characteristics and pathology with lesion detection and conspicuity. Eur J Radiol 2016;85:815-23.

4. Le Bihan D. Diffusion, perfusion and functional magnetic resonance imaging. J Mal Vasc 1995;20:203-14.

5. Chen X, Li WL, Zhang YL, et al. Meta-analysis of quantitative diffusion-weighted MR imaging in the differential diagnosis of breast lesions. BMC Cancer 2010;10:693.

6. Tsushima Y, Takahashi-Taketomi A, Endo K. Magnetic resonance (MR) differential diagnosis of breast tumors 
using apparent diffusion coefficient (ADC) on 1.5-T. J

Magn Reson Imaging 2009;30:249-55.

7. Horvat JV, Davila BB, Helbich TH, et al. Diffusionweighted imaging (DWI) with apparent diffusion coefficient (ADC) mapping as a quantitative imaging biomarker for prediction of immunohistochemical receptor status, proliferation rate, and molecular subtypes of breast cancer. J Magn Reson Imaging 2019;50:836-46.

8. Yamada T, Kanemaki Y, Okamoto S, et al. Comparison of detectability of breast cancer by abbreviated breast MRI based on diffusion-weighted images and postcontrast MRI. Jpn J Radiol 2018;36:331-9.

9. Grimm LJ, Soo MS, Yoon S, et al. Abbreviated screening protocol for breast MRI: a feasibility study. Acad Radiol 2015;22:1157-62.

10. Moschetta M, Telegrafo M, Rella L, et al. Abbreviated Combined MR Protocol: A New Faster Strategy for Characterizing Breast Lesions. Clin Breast Cancer 2016;16:207-11.

11. Bickelhaupt S, Laun FB, Tesdorff J, et al. Fast and Noninvasive Characterization of Suspicious Lesions Detected at Breast Cancer X-Ray Screening: Capability of Diffusion-weighted MR Imaging with MIPs. Radiology 2016;278:689-97.

Cite this article as: Shao Z, Liu P, Zhang S, Lu H. Abbreviated protocol combining quantitative diffusion-weighted imaging: a new strategy increasing diagnostic accuracy for breast magnetic resonance imaging? Gland Surg 2021;10(9):2705-2714. doi: $10.21037 / \mathrm{gs}-21-463$
12. Sagara Y, Mallory MA, Wong S, et al. Survival Benefit of Breast Surgery for Low-Grade Ductal Carcinoma In Situ: A Population-Based Cohort Study. JAMA Surg 2015;150:739-45.

13. Ponti A, Ronco G, Lynge E, et al. Low-grade screendetected ductal carcinoma in situ progresses more slowly than high-grade lesions: evidence from an international multi-centre study. Breast Cancer Res Treat 2019;177:761-5.

14. Hamilton LJ, Evans AJ, Wilson AR, et al. Breast imaging findings in women with BRCA1- and BRCA2-associated breast carcinoma. Clin Radiol 2004;59:895-902.

15. Schrading S, Kuhl CK. Mammographic, US, and MR imaging phenotypes of familial breast cancer. Radiology 2008;246:58-70.

16. Onega T, Weaver DL, Frederick PD, et al. The diagnostic challenge of low-grade ductal carcinoma in situ. Eur J Cancer 2017;80:39-47.

17. Lehotska V, Rauova K, Vanovcanova L. Multiparametric MRI analysis of morphologico-functional features of DCIS - correlation with grade of nuclear atypia. Neoplasma 2018;65:389-97.

(English Language Editors: B. Meiser and J. Chapnick) 